\title{
Dynamical model for competing opinions
}

\author{
S. R. Souza ${ }^{1,2, *}$ and S. Gonçalves ${ }^{2, \dagger}$ \\ ${ }^{1}$ Instituto de Física, Universidade Federal do Rio de Janeiro, Cidade Universitária, Caixa Postale 68528, Rio de Janeiro 21941-972, Brazil \\ ${ }^{2}$ Instituto de Física, Universidade Federal do Rio Grande do Sul, Avenida Bento Gonçalves 9500, Caixa Postale 15051, Porto Alegre \\ 91501-970, Brazil
}

(Received 7 January 2012; revised manuscript received 20 March 2012; published 3 May 2012)

\begin{abstract}
We propose an opinion model based on agents located at the vertices of a regular lattice. Each agent has an independent opinion (among an arbitrary, but fixed, number of choices) and its own degree of conviction. The latter changes every time two agents which have different opinions interact with each other. The dynamics leads to size distributions of clusters (made up of agents which have the same opinion and are located at contiguous spatial positions) which follow a power law, as long as the range of the interaction between the agents is not too short; i.e., the system self-organizes into a critical state. Short range interactions lead to an exponential cutoff in the size distribution and to spatial correlations which cause agents which have the same opinion to be closely grouped. When the diversity of opinions is restricted to two, a nonconsensus dynamic is observed, with unequal population fractions, whereas consensus is reached if the agents are also allowed to interact with those located far from them. The individual agents' convictions, the preestablished interaction range, and the locality of the interaction between a pair of agents (their neighborhood has no effect on the interaction) are the main characteristics which distinguish our model from previous ones.
\end{abstract}

DOI: 10.1103/PhysRevE.85.056103

PACS number(s): 05.65.+b, 89.75.-k, 87.23.Ge

\section{INTRODUCTION}

Statistical mechanics has turned out to be quite successful in modeling many systems whose interactions are, in principle, much more complex than those traditionally studied in physics as, in many cases, the systems are made up of agents which are endowed with intelligence and therefore the interaction between them depends on their decisions [1-3]. Nevertheless, simple statistical models have been developed for describing social systems [1-7], the economy [8-11], etc. Despite the great complexity of such real systems, their main properties can be reproduced by simple models which retain their underlying features, and the explanation for this fact is related to the concept of universality $[12,13]$.

A great deal of effort has been devoted to developing models for describing the properties of systems made up of agents with competing opinions $[1,4-7,14-20]$. This is of great relevance as human conflicts very often arise from the simultaneous existence of incompatible opinions in populations. Different systems, such as hierarchical societies [14] or democratic ones [16], where the agents follow the opinion of the local majority within a group, have, for instance, been investigated. Most of these models allow the agents to assume only one of two possible opinions. Such spin-flip models are representative of many real situations which offer only two possibilities and therefore are also of great interest $[1,17,18]$, along with the similarities with other physical systems. Axelrod's model for dissemination of culture is particularly different from the rest because the agents interact only if some degree of coincidence exists between them [5-7].

The evolution of scientific paradigms has been recently modeled in Ref. [4], where agents located on a square lattice can adopt one of the existing ideas or, occasionally,

\footnotetext{
*srsouza@if.ufrj.br

${ }^{\dagger}$ sgonc@if.ufrgs.br
}

create new ones. In contrast to the models mentioned above, there is no limitation on the number of ideas that may be created, except that an agent never returns to an idea it has previously abandoned. The influence of the neighborhood on the interaction between the agents, however, is a feature shared with the models just mentioned. Therefore, the interaction may be seen as a kind of mean field. The slow decline of old concepts and the quick adoption of new ones are the main characteristics of the model. The authors of Ref. [4] find that the dynamics naturally leads to the replacement of old concepts by a new paradigm, which dominates for a certain period of time, until it is gradually replaced by new ideas.

Inspired by that work, we have developed an opinion model where agents are placed at the vertices of a regular lattice and interact only with those located within a certain range, defined by a model parameter, in contrast to Ref. [4] where no such restriction is imposed. In this way, we allow the agents to interact with those located beyond their first neighbors but restrict the interaction to a preestablished range. In our model, each agent has an opinion and its degree of conviction. Different from many other models, such as those developed in Refs. [4,16-20], for instance, the interaction between two agents is strictly local, in the sense that it relies only on the interacting agents' properties, i.e., on their individual opinions and convictions. Therefore, during the interaction between a pair of agents, the fact that one of them may eventually be surrounded by a competing opinion or supported by its companions has no influence on the outcome of the interaction. This is one of the main characteristics that make our model very different from the previous ones briefly mentioned above, whose interaction is based on the properties of the agents' neighborhoods. During the interaction only the interacting agents' convictions are affected. If the conviction of one of the agents reaches a certain lower bound, then its opinion changes to that of the opponent. We therefore take into account the difficulty in persuading someone who has a strong conviction. 
In such a case, it is necessary to change that agent's beliefs prior to the acceptance of the new idea.

The dynamics of the opinion distribution in populations is then studied in the framework of this model, and the remainder of this work is organized as follows. In Sec. II we give a detailed description of the model. The results are presented and discussed in Sec. III, and concluding remarks are given in Sec. IV.

\section{THE MODEL}

The system is built on a regular mesh of $N_{x}$ horizontal and $N_{y}$ vertical lines, with periodic boundary conditions. One agent is placed at each vertex, and an opinion $O_{i}$, among the $N_{o}$ possible ones, is randomly assigned to the $i$ th agent along with a positive integer $C_{i}$, which corresponds to its degree of conviction. An agent located at vertex $(k, l)$ interacts with any of the neighbors located in vertices $\left(k^{\prime}, l^{\prime}\right) \neq(k, l)$, where $k^{\prime}=k-r, k-r+1, \ldots, k+r-1, k+r$ and $l^{\prime}=l-r, l-$ $r+1, \ldots, l+r-1, l+r$. The range $r$ is one of the model parameters. Thus, each agent has $4 r(r+1)$ neighbors. At each step of the dynamics, the following occur.

(a) An agent $i$ is sampled with probability proportional to $C_{i}$, and one of its neighbors is randomly selected for interaction, as described below. By doing so we assume that the agent's activity is related to its conviction.

(b) With probability $\alpha$, another agent is randomly selected among the others, and its opinion changes to any of the $N_{o}$ possible ones, including its own. This procedure represents the replacement of the agent by death or substitution due to departure from its neighborhood. Its new conviction is selected between 1 and the maximum existing value so as not to introduce any bias into the system.

In step (a), nothing is done if the agents have the same opinion, and one then proceeds to step (b).

Two agents $i$ and $j$ may interact only if their opinions are different. In this case, they do it with probability

$$
p=\exp \left[-\lambda\left(C_{\min } / C_{\max }\right)^{2}\right],
$$

where $C_{\min }\left(C_{\max }\right)$ is the minimum (maximum) between $C_{i}$ and $C_{j}$ and $\lambda$ is a parameter which is chosen so as to minimize the interaction between agents for which $C_{i} \approx C_{j}$. This is because it is very unlikely that a leader would be influenced by another competing leader (two agents with large and similar values of $C$ ). On the other hand, if a leader (large $C$ value) meets an ordinary agent (small $C$ value) who has a different opinion, the latter is very likely to be convinced by the former. The functional form chosen in this work aims to introduce these features into the model. If the agents interact, with probability $C_{i} /\left(C_{i}+C_{j}\right)$, the conviction $C_{i}$ is increased by one unit, and $C_{j}$ decreases by $\left|C_{i}-C_{j}\right|$. Otherwise, $C_{j}$ increases by one unit, and $C_{i}$ decreases by $\left|C_{i}-C_{j}\right|$. If $C_{j} \leqslant 0\left(C_{i} \leqslant 0\right)$, then its opinion changes to that of agent $i(j)$, and $C_{j}\left(C_{i}\right)$ is set to unity.

\section{RESULTS AND DISCUSSION}

At the initialization stage, an opinion $1 \leqslant O_{i} \leqslant N_{o}$ and the conviction degree $1 \leqslant C_{i} \leqslant 10$ [21] are randomly selected and assigned to the $i$ th agent. The system then evolves during at

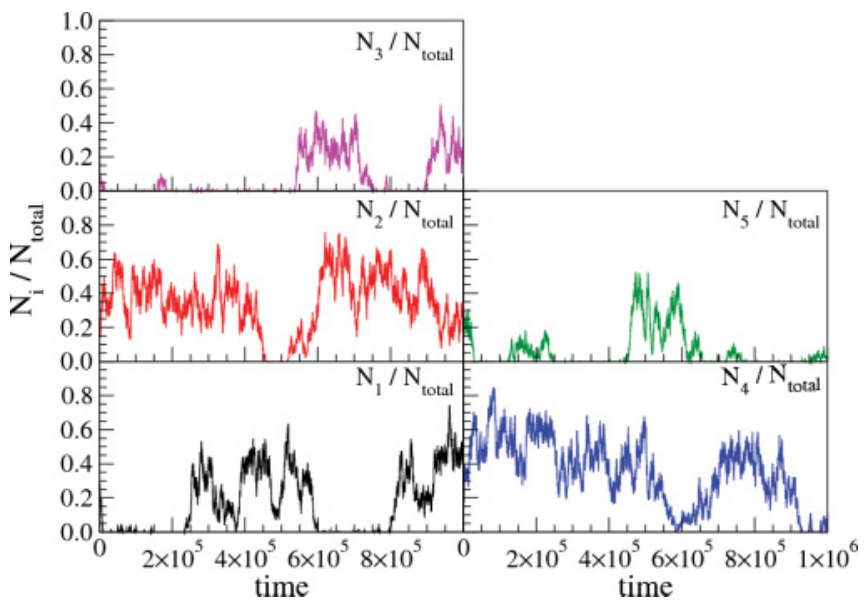

FIG. 1. (Color online) Fractions of the population $\left(N_{i} / N_{\text {total }}\right)$, with opinion $1 \leqslant i \leqslant N_{o}=5$, as a function of time. The curves show the results for $r=1, \lambda=1.0, N_{o}=5, \alpha=1.0 \times 10^{-6}$, and $N_{x}=N_{y}=100$. For details, see the text.

least $10^{8}$ steps. A full step corresponds to the time interval during which $N_{x} \times N_{y}$ intermediate steps, as explained in steps (a) and (b) in Sec. II, take place.

We start by examining the time evolution of the populations $N_{i}\left(1 \leqslant i \leqslant N_{o}\right)$ of groups with the same opinion, normalized by the total number of agents,

$$
N_{\text {total }}=\sum_{i=1}^{N_{o}} N_{i} .
$$

The results obtained using $\lambda=1.0, r=1, N_{o}=5, \alpha=1.0 \times$ $10^{-6}$, and $N_{x}=N_{y}=100$ are shown in Fig. 1 . For clarity, we have restricted the time scale to $10^{6}$, in spite of having carried out simulations up to much larger times, as just mentioned. One sees that no opinion dominates the dynamics. The opinions coexist in different proportions, and one notices that, very often, there is one opinion which is much more popular than the others. Its dominance lasts for a relatively short time, and the popular opinion is replaced by not so popular ones. As a matter of fact, only a small number of opinions are effectively disseminated through the system, the others being a small perturbation most of the time. During the time evolution, the most popular opinions are replaced by the unpopular ones, but few opinions dominate the population at the same time.

This conclusion is independent of the number of possible opinions $N_{o}$, as one sees in Fig. 2, which shows the dynamics of the three largest populations (normalized by $N_{\text {total }}$ ). More specifically, at time step $t$, the indices $i$ of the opinions are reordered in descending order of population $N_{i}(t)$, and the first three groups $\left(M_{1}>M_{2}>M_{3}\right)$ are selected. The calculations have been carried out for $N_{o}=50$ for both short and long ranges, i.e., $r=1$ and $r=5$, and are respectively displayed in the left and right panels of Fig. 2. The bottom three panels on each side show the values of $M_{1} / N_{\text {total }}, M_{2} / N_{\text {total }}$, and $M_{3} / N_{\text {total }}$ (as indicated in each plot). It is clear from these results that, although the composition changes, only a very few opinions are actually adopted by the agents. As in the previous case, opinions rise and fall during the dynamics. Furthermore, from the top panels, which exhibit the sum of the three largest 


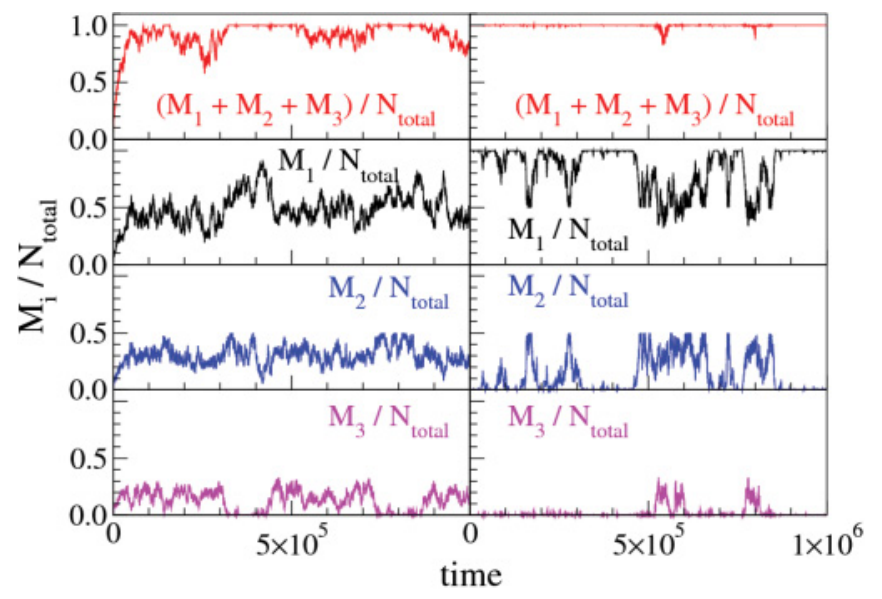

FIG. 2. (Color online) Populations of the most abundant opinions $\left(M_{1}>M_{2}>M_{3}\right)$ at each step divided by the total number of agents $N_{\text {total }}$ as a function of time. The left panels show the results for short range interaction $r=1$, whereas $r=5$ has been used in the calculations shown in the right panels. In both cases, $N_{o}=50$ opinions are allowed, and the remaining parameters of the simulations are $\lambda=1.0, \alpha=1.0 \times 10^{-6}$, and $N_{x}=N_{y}=100$. For details, see the text.

populations, normalized by the total number of agents, one sees that this sum nearly exhausts the normalization almost all the time. Therefore, the use of a fixed number of opinions should not be seen as a limitation of the model as the system naturally eliminates most of the competing opinions and very few of them effectively play any part in the dynamics at the same time. A comparison between the left and right panels of Fig. 2 shows that the effect of the interaction range $r$ on this observable is mainly quantitative, although there seems to be a tendency for the dominance of two groups. We have checked that, when varied within the range $1.0 \leqslant \lambda \leqslant 2.0$, the results' dependence on $\lambda$ turned out to be weak. Since this conclusion holds for other observables, such as the size distribution (discussed below), we adopt $\lambda=1.0$ in all the calculations. The parameter $\alpha$ has also been varied, and its effect on the composition of the opinions was found to be quantitative rather than qualitative. The role played by $\alpha$ on other properties of the system is further investigated below.

We now turn to the size distribution of clusters made up of neighbors holding the same opinion. The system configuration is analyzed at every $10^{3}$ full steps, and the time average is thus performed. The results are exhibited in Fig. 3 for different values of the range $r$ and system sizes (as indicated in each plot). Two agents belong to the same cluster if they have the same opinion, and their grid coordinates obey $(k, l)-\left(k^{\prime}, l^{\prime}\right)=$ $( \pm 1,0),(0, \pm 1)$, or $( \pm 1, \pm 1)$. The results clearly show that the size distribution is very sensitive to the interaction range $r$ and the system sizes. For cluster sizes up to $10 \%$ of the total system, the distribution becomes steeper as $r$ increases, whereas the development of a big cluster, of approximately the size of the total system, becomes more and more pronounced. Since agents interact only if they do not have the same opinion, the borders between clusters are the regions of strong activity. For short range interactions, only agents which are located very close to the borders are allowed to interact. Therefore,

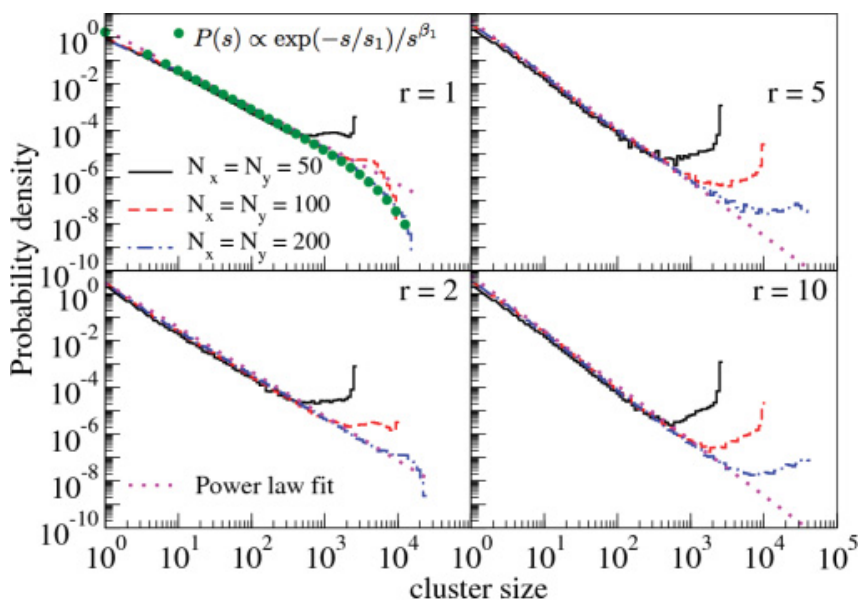

FIG. 3. (Color online) Size distribution of clusters made up of neighbor agents which have the same opinion. Some of the model parameters are listed on the panels, whereas the others correspond to $\lambda=1.0$ and $\alpha=1.0 \times 10^{-6}$. The power laws (dashed lines) are best fits to the results, whose exponents $\beta=1.65,1.90,2.30$, and 2.35 are respectively associated with $r=1,2,5$, and 10 . The solid circles in the top left panel correspond to $P(s) \propto \exp \left(-s / s_{1}\right) / s^{\beta_{1}}$, $s_{1}=3703.7$, and $\beta_{1}=1.65$. For details, see the text.

for small values of $r$, one should expect to observe compact groups of agents which share the same opinion. This should favor the appearance of medium size clusters. Indeed, large range values would lead to very diffuse borders and therefore to the disappearance of the coherence among the agents which are close to each other. In the limit of very large $r$ values, the connected agents would pervade the system, and the different groups would interpenetrate each other, as they would not be compact. This would enhance the appearance of large clusters during the dynamics, whose contributions to the size distribution may also be noticed in Fig. 3. Owing to the strict conservation laws, the multiplicity of small clusters should then decrease, and their size distribution would be steeper, as is also seen in Fig. 3.

This qualitative reasoning is confirmed by the results displayed in Fig. 4, where the spatial configuration of the clusters is shown at randomly selected moments. Distinct opinions are represented by different colors (shades of gray). One sees that compact groups are indeed formed for $r=$ 1 , whereas the clusters become more and more spatially diffuse as $r$ increases. Thus, our model predicts that long range interactions tend to destroy spatial correlations among opinions when consensus has not been reached and different opinions coexist in the system.

One may also notice in Fig. 3 that the size distribution of clusters whose size $s$ is smaller than $10 \%$ of the system size is fairly accurately approximated by a power law, i.e., $P(s) \propto$ $s^{-\beta}$, which is depicted by the dotted lines. The exponent varies with $r$ and corresponds to $\beta=1.65,1.90,2.30$, and 2.35 for $r=1,2,5$, and 10 , respectively. It should be noticed that the variation of $\beta$ is very small for $5 \leqslant r \leqslant 10$, which reveals that the power law exponent rapidly converges to an asymptotic value. Indeed, we have checked that, within the uncertainties of the fit, $\beta$ is very close to this limit at $r=10$. 

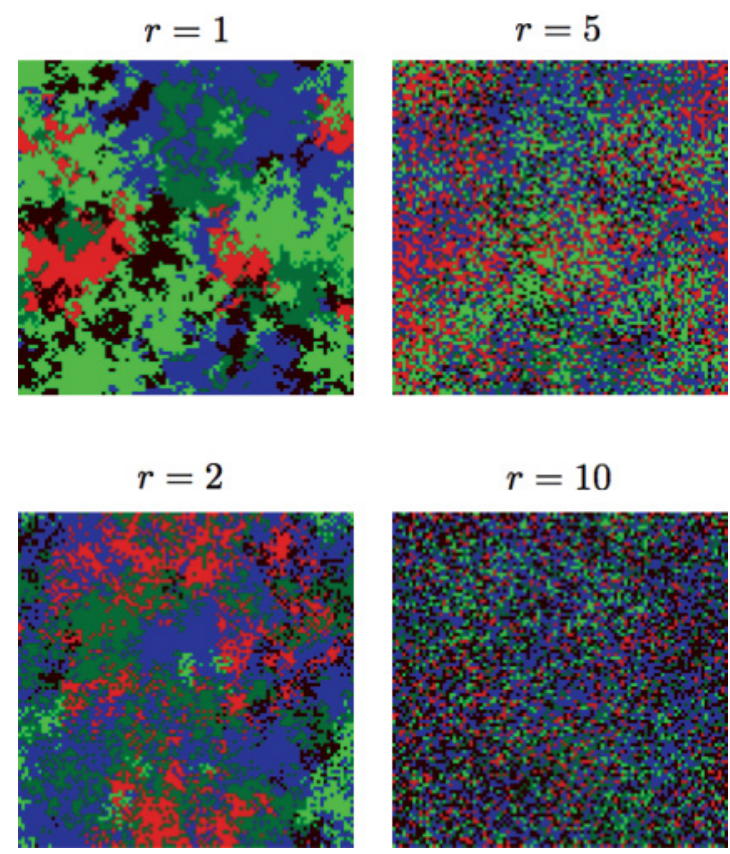

FIG. 4. (Color online) Spatial cluster distribution at randomly selected moments of the dynamics. Agents sharing the same opinion are depicted by the same color (shade of gray). The simulation has been carried out using $N_{o}=5, \lambda=1.0$, and $N_{x}=N_{y}=100$. For details, see the text.

In the different panels of Fig. 3 we compare the size distributions of different system sizes at fixed values of the range $r$. The results show that, for $r=1$, there seems to be a characteristic scale since the largest cluster formed during the dynamics does not scale with the system size. In fact, as shown by the solid circles in the top left panel of Fig. 3, the distribution for $r=1$ and $N_{x}=N_{y}=200$ is accurately described by $P(s) \propto \exp \left(-s / s_{1}\right) / s^{\beta_{1}}, s_{1}=3702.7$, and $\beta_{1}=1.65$, which has a clear exponential cutoff at $s=s_{1}$. The situation nevertheless changes very quickly for larger $r$ values, and such and exponential cutoff is not clearly seen for $r=2$. More precisely, the power law regime extends to larger sizes as the total system size increases for $r \geqslant 2$. Actually, there is a range value, between 2 and 5, for which the power law regime is an adequate description of the size distribution, except for very large clusters since finite size effects have to be considered for those clusters. Thus, the dynamics leads to two distinct scenarios. For small $r$, compact clusters are formed and occasionally amalgamate and form a very large one. Owing to the locality of the coalescence process (for small $r$ the interactions take place at the clusters' borders), this happens very rarely since it requires strong spatial correlations, hindering the formation of very large clusters. Then, this process leads to the existence of a characteristic scale. On the other hand, when large $r$ values are used, the coalescence extends through much larger areas due to the spread of the clusters. It therefore makes it easier for correlations to propagate through the entire system. Thus, the present model predicts that the size distribution of not too large clusters is fairly well described by a power law if the interaction between the agents is not restricted to their contiguous neighborhood.
The power law behavior of the size distribution suggests that the system reaches a critical state during the dynamics, in close analogy to critical phenomena in equilibrium statistical mechanics. In the latter case, the system is driven to a critical state by the external tuning of one its parameters, such as its temperature. The situation is, nonetheless, distinct from the present one since, as illustrated by Figs. 1 and 2, the configuration of the system is always changing and no static state is ever reached due to the noise introduced in step (b) of Sec. II. Furthermore, here it is the system itself that adjusts its internal degrees of freedom to reach the critical state and to remain close to it. This is called self-organized criticality (SOC) and has been discussed in many different places [12,22-26]. It has also been observed in a large diversity of systems [12,22-30]. The asymptotic value of the power law exponent of the size distribution shown above lies within the values obtained with the random site percolation (RSP) whose exponent ranges from 2.05 for two-dimensional lattices [13] or $2.186 \pm 0.002$ for three-dimensional lattices [31] up to 2.5 for the Bethe lattice [13]. This exponent is larger than that obtained with a nonconsensus opinion (NCO) model [17] whose power law exponent of the size distribution is $1.89 \pm 0.01$, which is very close to the value of $1.90 \pm 0.01$, obtained with the invasion percolation with trapping (TIP) $[32,33]$. These values reveal that our model and the NCO model belong to different universality classes, the latter being associated with that of the TIP. Indeed, except for short range interactions $r=2$, the power law exponents obtained with our model are larger than that predicted by the NCO model. However, in spite of lying within the range of exponents obtained with the RSP for $r>2$, further investigations are necessary to better understand the universality class of our model, which is beyond the scope of the present study.

At the critical state, the system has no characteristic length, whose consequences manifest themselves as size distributions that follow power laws. Similar long range correlations should also be observed in time when the system is near the critical point [12]. In order to investigate whether this property is actually present in our model, Fig. 5 shows the distribution of time intervals $\Delta t$ during which an opinion stays as the most popular one. The bottom panel of Fig. 5 displays the distribution of $\Delta t$ for different values of the range (see the legend). The power law fit $P(\Delta t) \propto \Delta t^{-\gamma}$ to the results reveal that this is a fairly accurate representation of the distribution for $\gamma=1.5$, even for small values of $r$, since the power law behavior is followed by the simulation results through more than three decades. Deviations from the power law are observed only at large values of $\Delta t$.

Up to this point, we have not thoroughly investigated the role played by the parameter $\alpha$, which regulates the frequency with which an agent randomly changes its opinion. It contributes with noise, which prevents the system from freezing when consensus is reached. In this sense, the model strongly relies on this parameter to ensure an interesting dynamics. The top panel of Fig. 5 illustrates the effects of $\alpha$ on the distribution of $\Delta t$ for $r=10$. We have focused on this value since the bottom panel of Fig. 5 shows that the dependence of $\Delta t$ on $r$ is weak. One sees that the validity of the power law fit is more and more impacted by the increasing amount of noise (larger values of $\alpha$ ) which is introduced into the dynamics. 


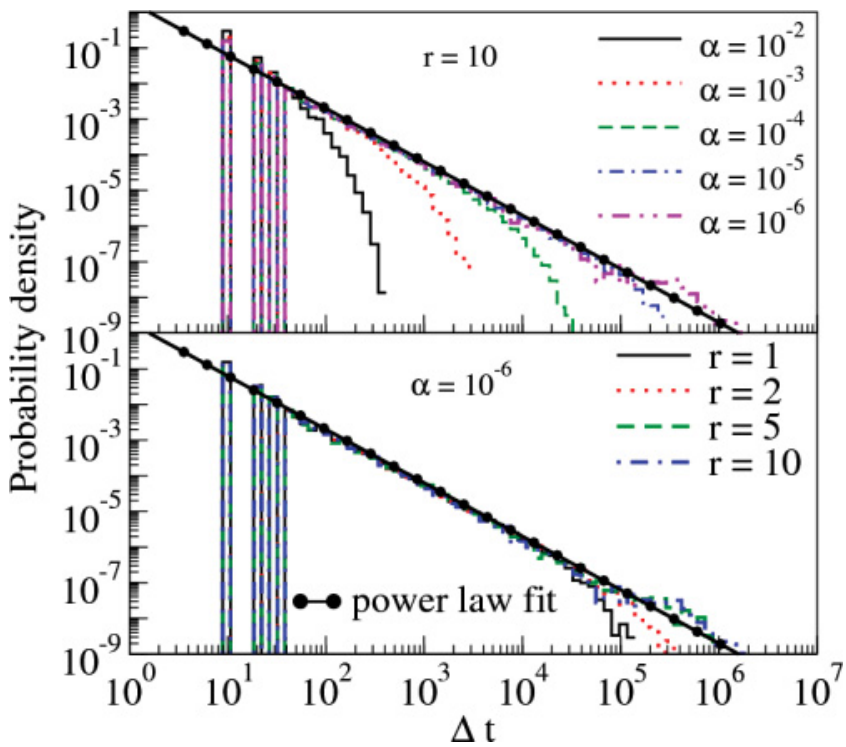

FIG. 5. (Color online) Distribution of time intervals during which an opinion remains the most popular one for different values of the parameters. The power law fit $P(\Delta t) \propto \Delta t^{-\gamma}$ to the simulation results gives $\gamma=1.5$. The parameters employed in the model simulations are listed on the figure legends, except for $\lambda$ and the system size, which are $\lambda=1.0$ and $N_{y}=N_{x}=100$. For details, see the text.

These results suggest that $\alpha \lesssim 10^{-5}$ does not affect the time interval distribution, at least for the $N_{x}=N_{y}=100$ system used in the simulations.

Similar conclusions can be drawn from the results displayed in the bottom panel of Fig. 6, which exhibits the cluster size distribution for different values of $\alpha$. Once more, for $N_{x}=N_{y}=100$, we find that the distribution is insensitive to $\alpha$ only if the noise is kept at a very low level, i.e., $\alpha \lesssim 10^{-5}$. The top panel of Fig. 5 and the bottom panel of

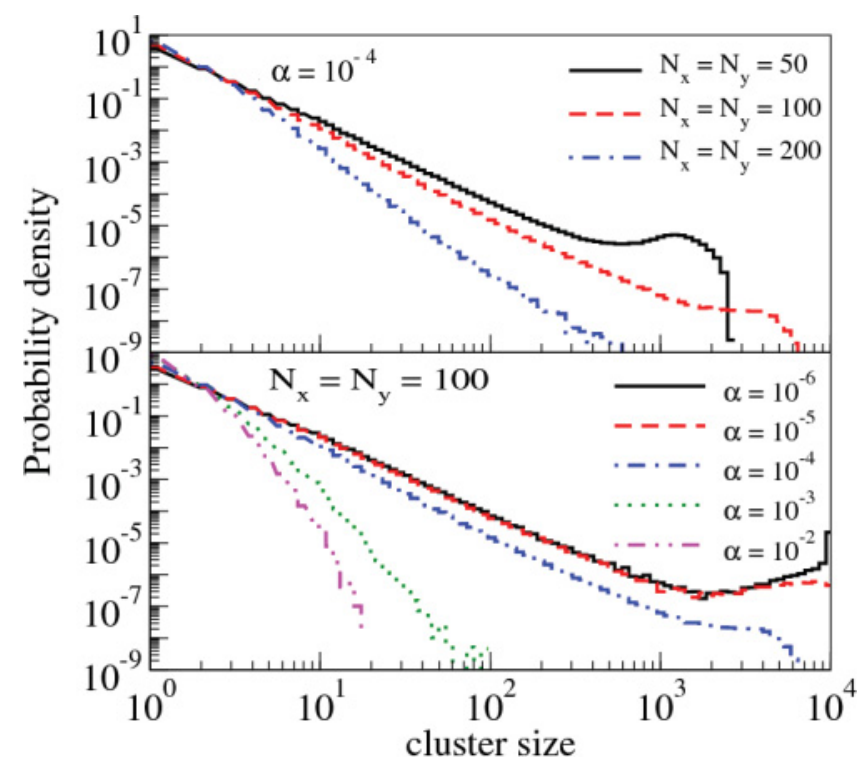

FIG. 6. (Color online) Cluster size distributions for different values of the parameter $\alpha$ and system sizes. The parameter values not listed on the legends are $\lambda=1.0$ and $r=10$. For details, see the text.

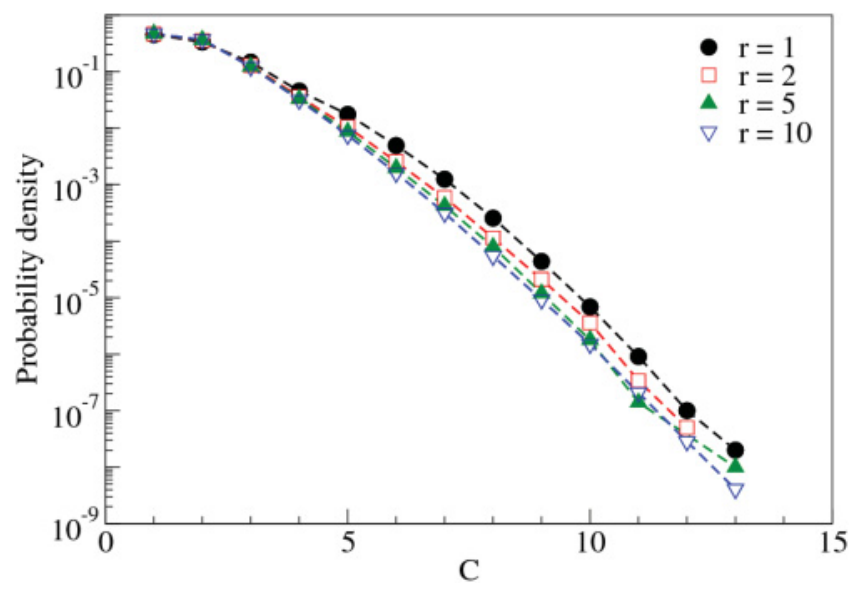

FIG. 7. (Color online) Time averaged conviction distribution. The parameters not listed in the legend are $N_{o}=50, N_{x}=N_{y}=100$, and $\lambda=1.0$. For details, see the text.

Fig. 6 clearly show that there is a transition from short range correlation (exponential decay), both in time and space, to long range correlation (power law behavior) as $\alpha$ decreases. This is because spatial correlations and long term memory gradually deteriorate as more and more noise is introduced into the system.

However, the results exhibited in the top panel of Fig. 6 clearly show that, although the cluster size distribution of not too large clusters is fairly well approximated by a power law, the exponent is very sensitive to the total system size. These results are at odds with those shown in Fig. 3 for $r=10$, from which one observes a clear independence from the total system size. Thus, it turns out that if too much noise is added to the dynamics by the parameter $\alpha$, the independence from system size is lost. This means that, although noise is needed by the dynamics, too much noise destroys the scale invariance; i.e., the agents must keep their opinions for at least a short while in order to preserve spatial correlations. We have checked that similar conclusions hold for $\alpha=10^{-5}$. Since, on average, $N_{x} \times N_{y} \alpha$ agents randomly change their opinions at each step, one sees that there is no unique value of $\alpha$ that would ensure scale invariance for arbitrary system sizes since one may always find a size for which too much noise is added to the system at each time step, destroying the spatial correlations. This shortcoming may be avoided by redefining $\alpha$ as the total rate per step, i.e., proportional to $\left(N_{x} N_{y}\right)^{-1}$, so that the desired amount of noise is introduced into the system during the dynamics for any system size.

Since the agents' convictions play an important role in the dynamics, as they directly influence their resistance to the adoption of new paradigms, we also examine this quantity. Thus, Fig. 7 displays the time averaged conviction distribution for different values of $r$. As in real life, most of the agents have a low degree of conviction, and the system has very few leaders (large $C$ values); i.e., the distribution decays exponentially. As expected, for a given value of $C$, the distribution falls off as $r$ increases since longer range interaction allows the agents to encounter others with different opinions more often (as illustrated in Fig. 4, agents with the same opinion tend to be closer for small $r$ values and they do not interact). 


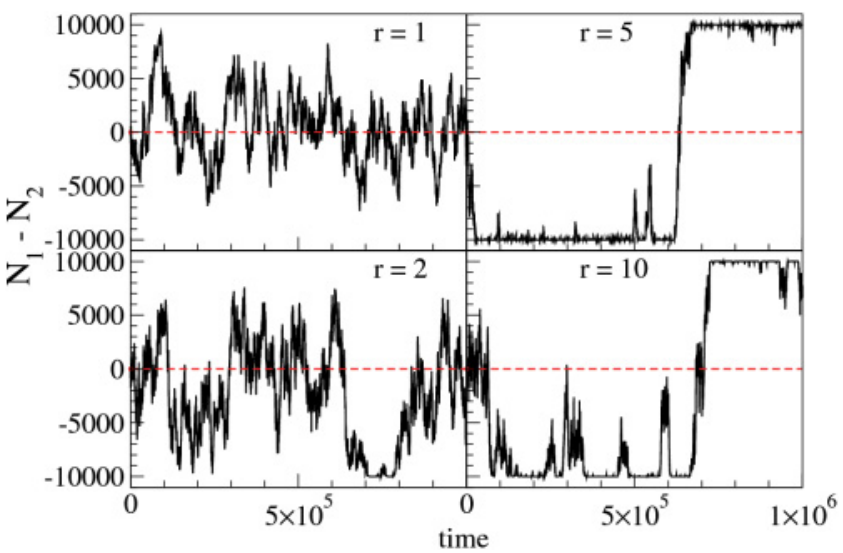

FIG. 8. (Color online) Time evolution of the difference between populations with opinions 1 and 2 . The parameters not listed on the plots are $N_{o}=2, N_{x}=N_{y}=100$, and $\lambda=1.0$. For details, see the text.

We finally examine the dynamics when only two opinions are allowed. The modeling of such systems is of great interest and has been extensively studied $[1,15,17-19]$ since there are many situations in real life where binary choices have to be made [1]. Furthermore, the above results, associated with Figs. 1 and 2, also suggest that this scenario should retain most of the properties of real systems. The results of the model simulation obtained with $N_{x}=N_{y}=100$ are displayed in Fig. 8, which exhibits the difference between the populations with opinion $1\left(N_{1}\right)$ and opinion $2\left(N_{2}\right)$. As in Refs. $[17,18]$, where the NCO model has been proposed and studied, our model allows for the dynamic coexistence of the two conflicting opinions with unequal population fractions. This is seen in the left panels of Fig. 8, which show the results for short range interactions between neighbors, i.e., $r=1$ and 2. This is in agreement with Ref. [17], which considers the interaction between the closest neighbors. For larger $r$ values, one sees that consensus is reached and it lasts for a long period. We have followed the dynamics during much longer time scales and confirmed this feature. Owing to the noise introduced by the random change of the agents' opinions, regulated by the parameter $\alpha$, the status quo does not last forever, and after being the overwhelmingly dominating opinion for a long period, its replacement occurs very quickly, and the other opinion becomes the consensus and so on. Therefore, our model predicts that a transition from nonconsensus to consensus occurs as the interaction among the agents changes from short to long range.

\section{CONCLUDING REMARKS}

We have developed a model for the dynamics of competing opinions which is based on the agents' degree of conviction and on the range of the interaction between them. It predicts that, even when many different opinions are allowed, only a very few of them are really in use by the agents during the dynamics. This is, in fact, observed in real life when, for instance, at the beginning of an election process, many candidates running for a political office start with not too different opportunities but, after a while, very few dominate the voters' preferences. The model also predicts that the size distribution of clusters, made up of agents which are located at contiguous spatial positions and share the same opinion, follows a power law. That distribution is reached independently of the initial conditions and does not need external tuning of any of its parameters, i.e., the dynamics leads to SOC [12,22-26], as long as the interaction between the agents is not restricted to too close neighbors. When only two opinions are allowed, the model leads to nonconsensus dynamics, which qualitatively agrees with the NCO model proposed in Ref. [17]. On the other hand, if the agents also interact with those who are located relatively far from them, consensus is quickly reached, and it lasts for a long time. The dominating opinion is occasionally replaced, but there is consensus almost all the time. Our model then provides a means to simulate many of the properties of real systems by changing a parameter which has a very simple interpretation on physical systems, i.e., the range of the interaction between the agents. It also contrasts with other models as the interaction between agents affects their conviction in first place and their opinions change only after their paradigms have been corroded. Furthermore, the interaction between a pair of agents is not influenced by their neighborhood, in contrast to most of the existing models.

\section{ACKNOWLEDGMENTS}

We would like to acknowledge CNPq, The MIT-Brazil Program/CNPq, a FAPERJ BBP grant, CNPq-PROSUL, FAPERGS, the joint PRONEX initiatives of CNPq/FAPERJ under Contract No. 26-111.443/2010, and CNPq/FAPERGS for partial financial support.
[1] C. Castellano, S. Fortunato, and V. Loreto, Rev. Mod. Phys. 81, 591 (2009).

[2] H. Ohtsuki, C. Hauert, E. Lieberman, and M. A. Nowak, Nature (London) 441, 502 (2006).

[3] S. Moss de Oliveira, P. M. C. de Oliveira, and D. Stauffer, Evolution, Money War and Computers (Teubner, Sttutgart, 1999).

[4] S. Bornholdt, M. H. Jensen, and K. Sneppen, Phys. Rev. Lett. 106, 058701 (2011).

[5] R. Axelrod, J. Conflict Res.41, 203 (1997).
[6] K. Klemm, V. M. Eguíluz, R. Toral, and M. San Miguel, Phys. Rev. E 67, 026120 (2003).

[7] J. C. González-Avella, V. M. Eguíluz, M. G. Cosenza, K. Klemm, J. L. Herrera, and M. San Miguel, Phys. Rev. E 73, 046119 (2006).

[8] L. Laloux, P. Cizeau, J.-P. Bouchaud, and M. Potters, Phys. Rev. Lett. 83, 1467 (1999).

[9] A. Charkraborti, I. M. Toke, M. Patriarca, and F. Abergel, Quant. Finance 11, 911 (2011).

[10] L. Kador, Phys. Rev. E 60, 1441 (1999). 
[11] R. Donangelo, A. Hansen, K. Sneppen, and S. R. Souza, Phys. A 348, 496 (2005).

[12] D. Stauffer, S. Moss de Oliveira, P. M. C. de Oliveira, and J. S. Sá Martins, Biology, Sociology, Geology by Computational Physicists (Elsevier, Amsterdam, 2006).

[13] D. Stauffer and A. Aharony, Introduction to Percolation Theory, 2nd ed. (Taylor \& Francis, Philadelphia, 2003).

[14] M. F. Laguna, S. R. Gusman, G. Abramson, S. G. Calves, and J. R. Iglesias, Phys. A 351, 580 (2005).

[15] M. S. de la Lama, I. G. Szendro, J. R. Iglesias, and H. S. Wio, Eur. Phys. J. B 51, 435 (2006).

[16] P. L. Krapivsky and S. Redner, Phys. Rev. Lett. 90, 238701 (2003).

[17] J. Shao, S. Havlin, and H. E. Stanley, Phys. Rev. Lett. 103, 018701 (2009).

[18] D. ben-Avraham, Phys. Rev. E 83, 050101 (2011).

[19] S. Galam, Europhys. Lett. 70, 705 (2005).

[20] R. Lambiotte and S. Redner, Europhys. Lett. 82, 18007 (2008).

[21] The upper bound for $C_{i}$ used in the initialization does not play a relevant role in the evolution since it is adjusted by the system during the dynamics.
[22] P. Bak, C. Tang, and K. Wiesenfeld, Phys. Rev. Lett. 59, 381 (1987)

[23] M. Paczuski, S. Maslov, and P. Bak, Phys. Rev. E 53, 414 (1996).

[24] P. Bak, C. Tang, and K. Wiesenfeld, Phys. Rev. A 38, 364 (1988).

[25] K. Sneppen, Phys. Rev. Lett. 69, 3539 (1992).

[26] P. Bak, How Nature Works: The Science of Self-Organized Criticality (Copernicus, New York, 1996).

[27] P. Bak and K. Sneppen, Phys. Rev. Lett. 71, 4083 (1993).

[28] R. Korsnes, S. R. Souza, R. Donangelo, A. Hansen, M. Paczuski, and K. Sneppen, Phys. A 331, 291 (2004).

[29] A. Chmel, V. N. Smirnov, and L. V. Panov, Ocean Sci. 3, 291 (2007)

[30] J. Sá-Martins and P. M. C. de Oliveira, Braz. J. Phys. 34, 1077 (2004).

[31] N. Jan and D. Stauffer, Int. Mod. Phys. C 9, 341 (1998).

[32] D. Wilkinson and J. F. Willemsen, J. Phys. A 16, 3365 (1983)

[33] S. Schwarzer, S. Havlin, and A. Bunde, Phys. Rev. E 59, 3262 (1999). 